\section{DE INDESEABLES A ILEGALES: UNA APROXIMACIÓN A LA IRREGULARIDAD MIGRATORIA}

\author{
Noelia González Cámara* \\ Instituto de Filosofía-CCHS (CSIC)
}

\section{FROM UNDESIRABLES TO ILLEGALS: AN APPROACH TO MIGRANT IRREGULARITY}

\begin{abstract}
The hipothesis of this paper is that irregular migration is not the simple consequence of entering in the territory of a State without authorization, however it is produced through legal and political measures. Migrations considered unauthorized do not have an static reference, the reference has evolved depending on sociohistorical circunstances. The objective is to denaturalize the use of the term "irregular migration" in light of historians and sociologists' understandings on the production of migrant irregularity. In order to achieve this aim I will engage in making some conceptual accuracies that clarify the different ways to designate this fenomenon and the realities it contains. Hence, it will be checked the way some flows have been identified as irregular in the United States, France, United Kingdom and Germany in the postwar period. Furthermore, an outline of its evolution will be made.
\end{abstract}

KEY WORDS: Irregular migration; migration policies; United States; France; United Kingdom and Germany.

Existen infinidad de nombres para designar a los miembros no deseados de una comunidad y el caso de los migrantes ${ }^{1}$ no autorizados no constituye una excepción. Las expresiones habituales evocan de forma constante la ausencia de alguna característica que se da por descontada en los ciudadanos. La negación y privación a la que aluden los términos empleados está referida fundamentalmente a la legalidad de su status, a la legitimidad de su presencia o al hecho de que sean bienvenidos o no por la sociedad. En cualquier caso, las expresiones han variado sustancialmente a lo largo del último siglo, desde términos con un marcado tinte peyorativo, como "indeseables", "ilegítimos", "inelegibles" o "ilegales", hasta expresiones más neutras empleadas en los años cincuenta y sesenta en las que se hacía hincapié en su carácter espontáneo o en el hecho de que llegaban por sus propios medios. En la actualidad, "migrante irregular", "clandestino", "no autorizado" e "indocumentado" se encuentran entre los términos más frecuentes.
RESUMEN: La hipótesis que vertebra este trabajo consiste en que la migración irregular no es la mera consecuencia de entrar sin autorización en un Estado sino un producto de medidas legales y políticas. Se sostendrá que las migraciones no autorizadas no tienen un referente estático, más bien dicho referente ha evolucionado en función de las circunstancias socio-históricas. El objetivo es, a la luz de las interpretaciones sobre la producción de irregularidad migratoria realizadas por historiadores y sociólogos, contribuir a la desnaturalización del uso del término "migración irregular". Para alcanzar este objetivo se propone realizar una serie de precisiones conceptuales que clarifiquen las distintas formas de designar este fenómeno, así como los supuestos que incluye. A partir de ahí se revisará cómo se ha llevado a cabo la identificación de determinados flujos como irregulares y su evolución en Estados Unidos, Francia, Reino Unido y Alemania tras la Segunda Guerra Mundial.

PALABRAS CLAVE: Migración irregular; política migratoria; Estados Unidos; Francia; Reino Unido y Alemania.

Las investigaciones sobre migraciones irregulares mantienen de forma recurrente el debate acerca de si tales términos pueden usarse como sinónimos o cuáles son sus límites semánticos. El hecho de que esta discusión siga abierta revela que no se trata de una mera discusión terminológica, sino que existe un problema epistemológico y conceptual más profundo (De Genova: 2002: 421-423). Siguiendo este planteamiento, en el primer apartado de este artículo se repasarán las categorías con las que se designan las migraciones no autorizadas y se realizará una aproximación analítica y tentativa a los contenidos que implica la irregularidad migratoria ${ }^{2}$. A partir de este breve análisis se mantendrá que intentar dar cuenta de los factores que configuran la irregularidad como si fuesen fijos e invariables en todas las sociedades y en todo momento histórico conduce a la naturalización de este fenómeno. La hipótesis que se va a emplear ya ha sido propuesta por diversos académicos (Dauvergne, 2008; De Genova, 2002: 424; 2004: 182; Düvell, 2010: 302; 2008: 
480; Ngai, 2004: 6) y consiste en que la migración irregular no es una realidad independiente o una simple consecuencia de entrar sin autorización en un Estado, sino que se produce en un contexto marcado por unas estructuras legales y políticas determinadas y en unas circunstancias socio-históricas particulares. Esto conduce, en el segundo apartado del artículo, a reconstruir la forma en la que se han gestado algunos de los usos de los conceptos con los que designa las migraciones no autorizadas. Se tratará de elaborar una panorámica de los acontecimientos que distintos historiadores y sociólogos han considerado claves en la historia de la producción de la irregularidad migratoria con la finalidad de poner de manifiesto que sus contenidos son dinámicos y que tanto la regularidad como la irregularidad se generan de forma legal, política y social. Para ello se propone centrar la atención en los casos de Estados Unidos, Francia, Reino Unido y la República Federal Alemana, todos ellos paises con una dilatada experiencia en la recepción de migración.

\section{Precisiones metodológicas preVias sobre UN CONCEPTO LÁBIL}

Como se ha adelantado, en la actualidad existen numerosas expresiones para designar aquellos flujos migratorios en los que la entrada, residencia o trabajo en el país de destino no han sido autorizados o en los que se han empleado medios fraudulentos para lograr los correspondientes permisos. Además, la economía semántica ha dado lugar a la designación de migrantes sin autorización de entrada y sin título de residencia regular directamente como "irregulares", "ilegales" o "clandestinos". Se sustantivizan, así, adjetivos que deberían hacer referencia a la situación administrativa de los migrantes, pues sólo las actividades, no las personas, pueden ser caracterizadas como ilegales (Schrover et al., 2008: 9). Los investigadores que se ocupan del estudio de las migraciones se han esforzado durante los últimos años en establecer precisiones entre los distintos términos debido a la utilización de los mismos como si fuesen sinónimos cuando, como ha señalado Frank Düvell, parten de puntos de referencia distintos: la ley, la posesión de documentación o la regularidad (Düvell, 2008: 484).

El debate más fuerte se ha polarizado entre el uso de términos que hacen referencia a la legalidad y aquellos otros que aluden a la regularidad. La carga peyorativa y despectiva fuerte que porta la expresión "migración ilegal" es uno de los argumentos que ha generado mayor rechazo (Dauvergne, 2008: 4; Fargues, 2009: 545; Ngai, 2004: xix; Nevins, 2002: 9). Se ha señalado, además, que la alusión de este término a la ley contribuye a la criminalización de los inmigrantes en situación irregular, cuando en buena parte de los Estados consiste únicamente en una falta administrativa y la mayoría de los migrantes en situación irregular no ha cometido ningún delito penal (Dauvergne, 2008: 16; Koser, 2005: 3; Nevins, 2002: 9). Otra consecuencia que se ha identificado como derivada de la designación de la migración no autorizada bajo la expresión "inmigración ilegal" consiste en la negación de la condición de individuo o personalidad legal del migrante (Koser, 2005: 3; Ngai, 2004: xix), puesto que la ilegalidad se asocia a procesos de exclusión, de restricción de derechos y de servicios, creando lo que Coutin ha denominado "espacios de no-existencia". Según Coutin (2000: 28), la creación y calificación de este campo como ilegal produce efectos nefastos, que en muy pocas ocasiones tienen consecuencias emancipatorias. Aún así, hay autores que prefieren el empleo del término migración y migrante "ilegal" frente a "irregular" (Dauvergne, 2008; Ngai, 2004; Schrover et al., 2008). En estos casos, se hace hincapié en las ventajas que ofrece la alusión directa de la expresión a la ley y a las condiciones que ésta establece para la migración, así como la concisión y popularidad del término (Dauvergne, 2008: 4). En este trabajo se argumentará que la producción de la irregularidad migratoria excede con mucho la esfera juridica, a diferencia de la tesis establecida por Dauvergne, y en este sentido se considera que la expresión "inmigración ilegal"3 no es plenamente adecuada. El hecho de que la entrada y residencia no autorizada no sea una actividad ilegal, sino una falta administrativa en muchos Estados contribuye a restar validez al término.

Las críticas vertidas a otros términos como "inmigrante irregular" o "inmigrante indocumentado" se basan en que mantienen cierta ambigüedad. Por un lado, el término "inmigración irregular" no establece con respecto a qué regulaciones son considerados irregulares los flujos $y$, por su parte, "inmigrante indocumentado" puede entenderse como la carencia de todo tipo de documentación identificativa, de documentación válida para la entrada y la residencia o incluso puede emplearse para hacer referencia a la entrada de aquellos migrantes que no ha sido conta- 
bilizada por las autoridades competentes (Koser, 2005: 5; Schrover et al., 2008: 10). En cualquier caso, el espectro de interpretaciones de estos términos es amplisimo: hay quienes consideran que la "migración indocumentada" sería un subgrupo de la "migración irregular o ilegal" en la medida en la que sólo hace referencia a las condiciones de entrada (Koser, 2005: 5; Fargues, 2009: 545; Ngai, 2004: xix; Strozza, 2004: 310), mientras que recientemente se está utilizando en un sentido laxo para designar también a aquellos migrantes que han incurrido en irregularidad por causas no relacionadas con la falta de documentación (De Genova, 2002: 420; Ngai, 2004: xix). La ventaja del término "inmigrante indocumentado" reside en que sus referentes son menos problemáticos y en que evita la alusión a la legalidad. Sin embargo, como han señalado Schrover, van der Leun, Lucassen y Quispel (2008: 10), cualquiera de los términos que se utilizan para designar la inmigración no autorizada puede adquirir las connotaciones negativas que se asocian al término "migración ilegal" y, por tanto, es mucho más apropiado prestar atención a cómo y en qué marco se desarrolla el debate sobre la irregularidad migratoria. En este sentido, se considera que lo más interesante del debate terminológico es que ha permitido desvelar problemas epistemológicos más profundos, como la reificación o naturalización a la que se han visto sometidas las migraciones no autorizadas (De Genova, 2002: 421-423).

Otros términos que cabe someter a examen son "migración extralegal" y "migración no autorizada". Este último puede entenderse como coincidente con "migración indocumentada" en el sentido de que ambos portan una referencia a la forma en la que se ha producido el acceso al territorio del Estado de acogida, aunque posteriormente el uso de ambos se ha extendido para designar cualquier tipo de migración en situación irregular. "Migración extralegal" no es un término frecuente y puede resultar equivoco en la medida en la que el prefijo extra- parece remitir a una situación que no está regulada por la ley, cuando la realidad que se designa se encuentra en situación diametralmente opuesta.

Por su parte, la peculiaridad de las expresiones "inmigrante clandestino" o "clandestino" e "inmigrante sin papeles" o "sin papeles" es que han surgido en contextos geográficos muy determinados y su uso se ha caracterizado por remitir a las migraciones no autorizadas que se han experimentado en Italia y Francia respectivamente. Ahora bien, en los últimos años estos términos han ganado popularidad, han sido traducidos a distintos idiomas y su empleo se ha extendido a otros Estados, aunque cabria someter a examen si se mantiene el mismo uso. Se ha documentado que el término clandestini se empleó por primera vez en Italia en 1992, en el contexto de aprobación de la Legge Martelli, mientras que la generalización de su uso data de 1995 (Düvell, 2010: 301). La designación de los migrantes no autorizados como "clandestinos" ha sido interpretado por parte de Kitty Calavita (2005: 135) como una reducción semántica en la que el referente está vinculado a actividades secretas y se convierte en sinónimo de una desviación peligrosa de la ley. Las connotaciones negativas del término son, por tanto, más que notables. Por su parte, la expresión sans papiers surgió en Francia en los años setenta en el contexto de las primeras movilizaciones contra la circulaire Marcellin y Fontanet (1972) a través de los encierros protagonizados por migrantes no autorizados. El término "sin papeles" porta una carga menos peyorativa que "inmigrante clandestino" al eludir la referencia a la ley y porque evita su identificación como actividad criminal. Si bien esta expresión es cada vez más frecuente en los discursos sobre irregularidad en los países del entorno europeo, sigue remitiendo de forma bastante especifica al caso francés, por lo que no se considera oportuno emplearlo de forma generalizada.

En el resto del trabajo se optará por utilizar las expresiones "migración irregular" y "migración no autorizada" en la medida en la que eluden uno de los peligros más importantes que acarrea el término "migrante ilegal": la criminalización de los flujos no autorizados. Ambas permiten incluir todos los supuestos de entrada y residencia fuera de la ley $y$, además, son menores las imprecisiones que se asocian a ellas. Queda por determinar cuál es el contenido que implica la irregularidad migratoria.

Al explicitar los elementos que configuran la irregularidad migrante mediante una exposición analítica es fácil incurrir en su naturalización, puesto que podría entenderse como un hecho dado, inmutable e independiente del contexto social, político y legal en el que se ha generado. Ahora bien, a través del estudio que se desarrollará en la próxima sección se dará cuenta de los procesos de ampliación y reducción a los que ha estado sujeta, así como los cambios terminológicos que ha experimentado. Aquí se proponen de forma tentativa el acceso, la residencia 
y el trabajo como las dimensiones básicas en torno a las cuales se ha definido hasta el presente la irregularidad migratoria (Düvell, 2008: 487; Fargues, 2009; Heckmann, 2004; Wihtol de Wenden, 1990). Un extranjero inmigrante está en situación administrativa irregular cuando carece de visado y de la documentación necesaria para acceder al territorio o cuando ha accedido de forma clandestina, bien eludiendo los controles de los puntos fronterizos 0 bien falsificando la documentación requerida. Un segundo caso de irregularidad migratoria se da cuando la residencia del inmigrante no ha sido autorizada. Esta situación puede ser consecuencia de una entrada irregular, aunque en los últimos años resultan más frecuentes los casos de extranjeros que entran en un Estado con visado de turista y que sobrepasan el período de estancia que establece dicho documento. En la literatura académica se denomina a los migrantes en esta situación con la expresión overstayers. El hecho de carecer de un permiso de trabajo se ha combinado con la forma de acceso y con la autorización de residencia como un criterio más de irregularidad, pero no existe acuerdo sobre si un trabajador extranjero con permiso de residencia podría ser considerado irregular por infringir los límites impuestos al ejercicio de una actividad laboral, como sostendrían Wihtol de Wenden (1990) o Düvell (2010). En este artículo se mantendrá que el criterio del trabajo por si solo no es determinante para definir la irregularidad, pues, como ha señalado López Sala (2003: 162), los migrantes que realizan trabajos no autorizados por su permiso o que lo hacen en la economía sumergida caen en el ámbito de la irregularidad laboral igual que cualquier nacional. No pueden ser considerados stricto sensu inmigrantes irregulares. En esta situación se encuentran los trabajadores inmigrantes que excedan las restricciones geográficas que establezcan sus permisos, que no respeten las limitaciones para trabajar en un sector profesional determinado o que trabajen por cuenta ajena cuando les esté vetado; asimismo, en muchos Estados los extranjeros que disfrutan de un visado de estudiante tienen limitado el permiso de trabajo a un máximo de horas por semana; la falsificación de documentación para trabajar en áreas distintas a las permitidas, así como la utilización de documentos legales de los que no se es titular para trabajar pueden ser consideradas una infracción de las condiciones establecidas para el trabajo, no para la residencia. En definitiva, tanto la violación de los requisitos de entrada como la no posesión del status de residente puede acarrear irregularidad migratoria en las distintas definiciones que los Estados han conformado de la misma, si bien hay que tener en cuenta que es muy común que se combinen y que también participe en alguna de sus múltiples variantes el trabajo irregular.

A partir de lo expuesto hasta ahora no se puede pasar por alto que lo que se designa bajo el rótulo "inmigración irregular" es un fenómeno amplio, complejo $y$, sobre todo, sumamente heterogéneo (Koser, 2005: 5-6; López Sala, 2003: 162). Además los límites entre el status regular e irregular son sutiles, pues es posible poseer un título de residencia regular pero trabajar sin el permiso correspondiente ${ }^{4}$. En este sentido, cabe considerar que la dicotomía regularidad/irregularidad migratoria es parcialmente falsa. Stoddard (1976: 23) y Fargues (2009: 546), entre otros, han señalado que en relación con la situación legal de los migrantes resulta más conveniente hablar de procesos dinámicos que de status legales claramente diferenciados y contrapuestos, en el sentido de que no son realidades estáticas sino que evolucionan, que uno sucede a otro y que la circularidad entre ambos es la tónica dominante. La debilidad del status del migrante regular producida por la amenaza continua de la pérdida del título de residencia legal marca una línea borrosa entre la regularidad y la irregularidad migratoria y hacen indiscernibles unos límites claros entre ambos.

El concepto de migración no autorizada no es, por tanto, autoevidente ni en la forma de designarlo ni en el contenido. La dicotomía regular/irregular se constituye de forma asimétrica, pues quién es migrante irregular no se define directamente en las legislaciones de los distintos Estados, sino que su significado se deduce de forma indirecta a partir de la definición de la migración regular. Por otro lado, existen buenas razones para sostener que la irregularidad migratoria no se puede definir única y exclusivamente en términos jurídicos. Brubaker (1992: 30) ha señalado cómo los de adentro y los de afuera de una comunidad, no necesariamente nacionales y extranjeros, pueden ser definidos formal o informalmente, bien a través de la aplicación de criterios establecidos y generales por parte de administradores especializados o bien mediante esquemas clasificatorios, prácticas tácitas, internas y no codificadas. Considero que este esquema puede aplicarse para estudiar la definición de la regularidad y la irregularidad migratoria, a diferencia de quienes sostienen que la migración irregular sólo existe en la medida que haya una ley que 
establezca condiciones de entrada y residencia (Dauvergne, 2008: 10; Sanz Díaz, 2004: 20). La postura que sostengo vendría apoyada por el hecho de que existen casos en los que se produce la representación de la presencia de determinados grupos nacionales como ilícita o directamente como ilegal independientemente de cuál sea su situación administrativa. El caso paradigmático se da en los Estados Unidos donde la irregularización de población de origen asiático y mexicano a través de distintas medidas y legislaciones que se han puesto en marcha desde finales del siglo XIX ha dado lugar a la identificación y construcción de estos grupos nacionales como ilegítimos, inasimilables y criminales, independientemente de que puedan disfrutar de un status de residencia regular o incluso de la nacionalidad estadounidense. De esta manera se han creado "ciudadanos extranjeros" que, aunque sean reconocidos como nacionales por la ley, son considerados extranjeros por la cultura y el Estado americanos (Ngai, 2004: 2). En este caso la distorsión entre los límites del adentro y del afuera de la comunidad política se hacen más que patentes, ya que no reconocer en ámbitos informales a mexicanos y asiáticos como miembros de la comunidad ha conducido a una exclusión efectiva y a su representación generalizada como "ilegales" (Nevins, 2002: 116).

Otro argumento que sustenta la afirmación de que la irregularidad migratoria no se puede restringir únicamente a su definición formal viene dado por el hecho de que del cruce irregular de una frontera no se sigue la categorización de dicha migración como irregular. El cruce irregular es un acto concreto mientras que la migración irregular supone la participación de procesos sociales mucho más amplios que confluyen para crear una condición social a partir de una infracción de la ley que puede definirse como falta administrativa o como delito. Es decir, las migraciones que llegan al territorio de un Estado sin atender a los cauces legales establecidos o que sobrepasan el tiempo establecido por un visado o permiso temporal de residencia no se constituyen inmediatamente como migraciones irregulares. En este sentido, se puede apuntar cómo Francia, a través de una ordenanza aprobada en $1945^{5}$, pasó a considerar delito la migración no autorizada sin que esta medida legal fuese acompañada de la aplicación generalizada de las penas previstas. De hecho, a partir de 1956 se puso en marcha un procedimiento mediante el cual se podía regularizar la situación de los trabajadores extranjeros que no habían entrado por los cauces establecidos por la ley. Dado que la sociedad francesa percibió social y políticamente su llegada como un beneficio en tanto que contribuía al crecimiento económico del país, elevaba los estándares de vida y lo hacía además a un bajo coste (Wihtol de Wenden, 1990: 33), su representación se realizó bajo la etiqueta de "migraciones espontáneas", en el sentido de que no llegaban reclutadas a través de la Oficina Nacional de Inmigración. En definitiva, la identificación de un tipo de movilidad como irregular va más allá de su definición técnica, puesto que existen grupos no definidos legalmente como ilegales pero que son asimilados socialmente a dicho status $y$, en el caso contrario, porque es posible que no se configure una condición social propia para quienes cometen una infracción de las leyes que tratan de gestionar las migraciones.

\section{Algunas reflexiones en torno a la producción DE IRREGULARIDAD MIGRATORIA}

Una de las cuestiones más interesantes sobre irregularidad migratoria que se han puesto de manifiesto en la literatura académica reciente es que la visibilización de los extranjeros inmigrantes irregulares no ha venido acompañada de la visibilización de la producción de dicha irregularidad a través de la ley. Como consecuencia de ello, no sólo no se ha sometido a estudio la irregularidad como condición socio-política, sino que además, y esto sería más grave, el desequilibrio ha favorecido la naturalización de la migración no autorizada como un fenómeno independiente del marco de un Estado y como una realidad propia (De Genova, 2002: 431). No obstante, esta afirmación es cuestionable, puesto que desde el campo de la historia y la sociología trabajos como los elaborados por Erika Lee (2002), Mae Ngai (2004) o Joseph Nevins (2002) han evidenciado los procesos a través de los cuales se ha gestado la irregularidad en el caso estadounidense y también puede encontrarse literatura al respecto para el ámbito europeo (entre otros: Calavita, 2005; Calavita y Suarez Navaz, 2003; Couper y Santamaria, 1984; Van Ejil, 2008). En cualquier caso, es necesario profundizar en el campo de estudio de la producción de la irregularidad a nivel social, político y legal y poner en relación las distintas fases y los contextos estudiados. La tarea a realizar consiste en desnaturalizar la irregularidad migratoria a través del cuestionamiento del supuesto carácter originalmente independiente de la 
inmigración irregular con respecto a las condiciones que lo han generado.

Existen dos estrategias posibles para llevar a cabo esta tarea. Una vía consistiría en presentar cómo la identificación de flujos migratorios como irregulares se ha producido únicamente en determinados Estados. Así, se trataría de poner de relieve la especificidad de la producción de irregularidad migratoria en tanto que resultado de los procesos acontecidos en unas sociedades particulares. Una segunda estrategia igualmente válida consistiría en analizar cómo evoluciona históricamente dentro de un determinado Estado la concepción de la migración irregular, cómo se amplian y reducen los supuestos que implican estancia o residencia no autorizada en el interior de un Estado. Dadas las constricciones de las que consta todo trabajo habrá que optar por una de las estrategias y aquí se optará por la segunda, dejando para un trabajo posterior la contrastación de experiencias de Estados que no han participado de la irregularización de migraciones con la experiencia de Estados que sí lo han hecho.

La tarea que se propone llevar a cabo no consiste en hacer la historia de la migración no autorizada ni la historia de los usos de los términos con los que se designa, sino la más modesta de realizar un repaso de la bibliografía reciente que ha estudiado la forma en la que se ha generado y ha evolucionado la producción de la irregularidad migratoria a partir de las experiencias de los Estados Unidos y los Estados de Europa occidental después de la Segunda Guerra Mundial y a partir de aquí hacer una serie de reflexiones críticas. La selección de estos Estados está justificada por el hecho de que los primeros usos del concepto inmigración ilegal e irregular tienen lugar en Estados Unidos y porque los desarrollos más notables se han elaborado, al menos hasta ahora, en ese país y en el área de Europa occidental.

\subsection{Antecedentes}

Los primeros registros del término "inmigrante ilegal" en los Estados Unidos datan de las décadas de 1920 y 1930, pero los estudios realizados han hecho hincapié en que el uso que entonces se hizo de dicha expresión no es equivalente al actual (Ngai, 1999: 91; 2004). Así, las interpretaciones que se han llevado a cabo han puesto de manifiesto que el significado de illegal alien era similar a términos utiliza- dos en décadas anteriores, como unwanted o undesirable immigration $^{6}$ y illegitimate o inelegible alien ${ }^{7}$, es decir, se utilizó la expresión "extranjero ilegal" para designar a los migrantes no autorizados como individuos indeseables e inasimilables en función de su raza, su origen nacional y los prejuicios asociados a estos criterios (Nevins, 2002: 95). En cualquier caso, el uso de tal expresión ha mantenido al menos un contenido de forma continuada hasta la actualidad: la identificación social de la migración irregular con la migración mexicana. Se considera que la producción de la irregularidad migratoria y su asimilación con los flujos procedentes de México excedió los cauces estrictamente legales, pues, como ya ha sido documentado (Ngai, 2003), este proceso no se derivó directamente de la aplicación de las leyes de cuotas de 1921 y 1924. La nueva normativa no establecía una cuota a los migrantes mexicanos, ni estaban excluidos de la ciudadanía. Se ha considerado, entonces, que la diferenciación entre las migraciones europeas y las mexicanas vino dada por la forma en la que se aplicaron estas leyes y otras medidas secundarias que se pusieron en marcha al calor de los ajustes que fue preciso implementar en el mercado de trabajo al aumentar las tasas de desempleo con la Gran Depresión (De Genova, 2004: 190). La supresión de las excepciones que permitian a los migrantes mexicanos cruzar la frontera para ser empleados como mano de obra en los estados del suroeste ${ }^{8}$, la imposición de visado en 1924, la puesta en marcha de distintos procedimientos administrativos que permitieron la regularización sólo de migrantes procedentes de Canadá y Europa ${ }^{9}$, las deportaciones en masa de cientos de miles de mexicanos, la creación de la Border Patrol y el ejercicio de los primeros controles en la frontera sur se han interpretado como medidas relevantes en la progresiva producción de irregularidad migratoria y su identificación con las migraciones procedentes de México (De Genova, 2003: 4; Nevins, 2002; Ngai, 2003; Samora, 1971: 40).

En el caso europeo, los primeros flujos migratorios que se clasificaron como "ilegales" datan igualmente de los años veinte y treinta pero no se registró en el contexto de migraciones laborales, sino por la movilidad de los refugiados y apátridas que generó la nueva situación política tras el fin de la Gran Guerra. El uso de la categoría "refugiado ilegal", como aquel refugiado que residía en el territorio habiendo transgredido las condiciones legales de entrada, no fue muy extendido según los estudios realizados (Van Ejil, 2008: 50). Y cuando se empleó, lo fue en el sentido 
de que eran refugiados no deseados, lo que marca también una distancia considerable con el uso que se hace hoy en día del término "ilegal". El estudio sobre el caso holandés realizado por Van Ejil destaca cómo la utilización del término ilegal en órdenes ministeriales del año 1938 hace referencia al cruce concreto de la frontera, al acto físico de atravesarla sin cumplir con las condiciones que establecía la legislación. Catalogar a los refugiados judíos que procedian de Alemania como ilegales suponía que fuesen considerados inmigrantes no deseados a los que no se debía dejar entrar en territorio holandés. No implicaba, por tanto, una categorización del fenómeno de la migración irregular (Van Ejil, 2008). La expresión "ilegal" también ha sido documentada por Caestecker en Bélgica a finales de los años treinta, concretamente en 1939, cuando la residencia no autorizada pasó a ser considerada delito. Si bien podía ser penada hasta con un año de cárcel, los cruces ilegales de la frontera fueron tolerados (Caestecker, 1998: 84-85; 2000:282). Este caso podría considerarse un ejemplo más de cómo de la estricta regulación legal de la irregularidad migratoria no se deduce la creación de la condición social del migrante irregular.

Otro antecedente en el uso del término "inmigrante ilegal" se ha situado en el Reino Unido en la década de los años treinta. Los flujos de migrantes judíos cuyo destino era Palestina fueron sujetos a un cupo anual de 10.000 personas a fin de no perjudicar las relaciones que mantenía el gobierno británico con la población árabe de Palestina, protectorado británico en aquel entonces (Sassen, 1996: 96). Así, se calificó sistemáticamente como "ilegales" a los migrantes judíos que no habían sido autorizados (Düvell, 2008: 480). En cualquier caso, el uso de este concepto no se hizo extensivo a otro tipo de migraciones y no se puede concebir como equivalente al actual.

En lo que se sigue se argumentará cómo la producción de ilegalidad migratoria que se llevó a cabo en los años veinte y treinta en Estados Unidos y Europa occidental con relación a los flujos de migrantes mexicanos y de refugiados, respectivamente, se disipó, al menos de forma temporal, tras la Segunda Guerra Mundial. En ambos lados del Atlántico norte se hizo necesario reclutar mano de obra extranjera para suplir las necesidades del sector económico y, además, en el caso europeo para la reconstrucción de países arruinados materialmente por la contienda.

\subsection{La producción de irregularidad en Estados Unidos}

Estados Unidos puso en marcha el Programa Bracero en 1942 como una medida extraordinaria para hacer frente a la escasez de mano de obra que se experimentó como consecuencia de la guerra y por la necesidad de trabajadores para el sector de la agricultura y la construcción de ferrocarriles ${ }^{10}$. No obstante, el número de visados de bracero expedidos no fue suficiente para satisfacer la demanda de trabajadores de la economía capitalista estadounidense y junto a los braceros llegaron migrantes que no cumplían con los requisitos establecidos, a quienes se llamó wetbacks o espaldas mojadas"11 (Calavita, 1992: 31 y ss.; De Genova y Ramos-Zayas, 2003: 5; Massey y Liang, 1989: 203; Samora, 1971: 43). La migración mexicana, que se había identificado como ilegítima e inasimilable durante los años treinta, tornaba de nuevo al territorio estadounidense, pero esta vez era bienvenida por los empresarios aunque no contase con la autorización pertinente.

El tratamiento que dieron las autoridades a esta migración en la década de los cuarenta es bastante ilustrativa de cómo se modifica la actitud hacia las migraciones en función de las necesidades del Estado de acogida. Hasta 1949 cuando un migrante mexicano no autorizado era interceptado por la policía no era deportado, sino trasladado hasta la frontera e introducido de nuevo en territorio estadounidense como bracero. Otra opción era que la policía condicionase al empleador para que contratase al migrante no autorizado como bracero y evitar, así, su traslado (Bustamante, 1977: 171; Calavita, 1995: 237; De Genova y Ramos-Zayas, 2003: 5). Este proceso, que se ha denominado drying out wetbacks o secado de espaldas mojadas (Calavita, 1992: 32; De Genova, 2004: 191), se dejó de aplicar debido al excedente de trabajadores mexicanos que se empezó a experimentar en la fase de recesión temprana a finales de dicha década. Estos acontecimientos se combinaron con la expansión de ideas que representaban a la migración mexicana como un problema, en el sentido de que ejercía presión a la baja en los salarios del suroeste y de que constituía un peligro para la salud pública (Calavita, 1992: 48; Nevins, 2002: 34; Samora, 1971: 50). Este proceso alcanzó su punto más álgido con la Operación Wetback, mediante la cual se expulsó a casi tres millones de migrantes mexicanos entre 1954 y 1955. Según De Genova (2004: 192), el ejercicio puntual y selectivo de estas deportaciones se convirtió en la base de la política de 
"puerta giratoria" estadounidense que permitía establecer un límite sutil entre la migración regular e irregular. Precisamente la "deportabilidad", no en cuanto deportación realmente efectuada, sino en cuanto potencial amenaza de deportación, desempeñó la labor disciplinante ${ }^{12}$ fundamental de estigmatizar a los trabajadores mexicanos $y_{\text {, }}$ sobre todo, de proporcionar al capitalismo estadounidense fuerza de trabajo extranjera flexible que se mantuviese en el límite entre no estar completamente excluida y no ser totalmente prescindible.

Así, la dependencia que el sector de la agricultura de los estados del sur experimentaba con respecto a la mano de obra mexicana no autorizada dio lugar a que aquél dirigiese quejas al Congreso por las deportaciones realizadas. Las reclamaciones fueron atendidas y se amplió el número de visados para la contratación de braceros hasta 1960. A partir de esa fecha se redujo a la mínima expresión el número de contrataciones legales y finalmente se puso fin al Programa Bracero en 1964. La conclusión del programa y la entrada en vigor de la nueva Immigration Reform Act en 1965 se han valorado como los dos factores claves que dieron lugar a una producción más rígida de la irregularidad migratoria mexicana. Si bien la nueva ley presentaba inicialmente numerosos procedimientos a través de los cuales los trabajadores mexicanos podían regularizar su situación, el establecimiento en 1976 de una cuota de 20.000 migrantes mexicanos anuales reducia las posibilidades de migrar de forma regular. Las migraciones de trabajadores mexicanos se siguieron desarrollando por la existencia de potentes redes migratorias asentadas y vínculos históricos consolidados. La existencia estribaba ahora en que la entrada en territorio estadounidense tenía lugar sin el status legal pertinente debido a la suspensión de los visados de bracero y por la introducción por primera vez de aquella cuota para las migraciones mexicanas (De Genova, 2004: 201).

Estas medidas estrictamente legales fueron acompañadas de un proceso de representación social de la migración mexicana como amenaza nacional. Así, a finales de los años sesenta se extendió a través de los medios de comunicación la idea de que la frontera sur estadounidense estaba "fuera de control" por las continuas llegadas de migrantes "ilegales" y se comenzó a hablar de una "invasión", "el problema de los espaldas mojadas" o de "crisis nacional" (Bustamante, 1977: 149-150; Gutiérrez, 1995: 188). La percepción de la pérdida de control en la frontera se debió más bien al intento de establecer control por primera vez en el territorio fronterizo con México, que al hecho de que realmente los cruces fronterizos hubiesen quedado al libre albedrío (Joppke, 1999: 31). El cambio de la situación económica a principios de los años setenta hizo que el debate calase en la opinión pública y la obsesión sobre el control de la frontera, a la que hasta entonces se había prestado atención puntualmente, se convirtiera en endémica y pasase a ser comprendida como una crisis nacional (Nevins, 2002: 62-63). Por otro lado, el hecho de que el Servicio de Inmigración y Naturalización identificase a los migrantes mexicanos como el $99 \%$ de los migrantes no autorizados que podian ser deportados y el incremento constante hasta 1978 de las acciones en este mismo sentido contribuyeron a sostener la idea de que los migrantes irregulares potenciales eran exclusivamente mexicanos (De Genova, 2004:199)

En los años siguientes, la búsqueda de un consenso político en torno a la gestión de las migraciones se implementó con la aprobación de una nueva ley en 1986, la Immigration Reform and Control Act, tras un largo período de debate político y social en el que se puso de manifiesto que uno de los objetivos principales era impedir la llegada de migración irregular. En este contexto, se ha de resaltar la postura que adoptó la Comisión a cargo de la elaboración de la ley, pues manifestó que Estados Unidos debía reconocer su papel y su responsabilidad en la creación del problema de la inmigración no autorizada y así hacía copartícipe a la sociedad para que tomase la iniciativa de atajar una situación que ella misma había generado (Joppke, 1999: 31-32; Nevins, 2004: 140). Esta actitud sería la piedra angular que sustentó posteriormente el proceso de regularización y la sanción de los contratistas que se hicieron efectivos con la ley de 1986.

La regularización de la inmigración no autorizada hizo disminuir sólo temporalmente la atención prestada a este fenómeno, pues a principios de los años 90 las cifras de irregulares volvieron a ser considerables y la actitud negativa hacia su presencia se hizo patente de nuevo en el aumento de las causas que daban lugar a la deportación en la Immigration Act (1990) y en el debate de la Proposition 187 de California. Tanto los desarrollos legislativos y las medidas políticas emprendidas, como las numerosas operaciones puestas en marcha en la frontera ${ }^{13}$, han endureci- 
do el tratamiento que se ha otorgado a la inmigración no autorizada y han contribuido a su criminalización. Si bien la idea más extendida es que esta tendencia se acentuó a partir de los atentados terroristas del 11 de septiembre, el recrudecimiento de las leyes de inmigración data de 1996 (Brotherton y Kretsedemas, 2008: 8). La consecuencia de la aplicación de la Illegal Immigration Reform, la Immigrant Responsability Act y la Antiterrorism and Effective Death Penalty Act fue el aumento del número de personas procesadas por infracciones relacionadas con la inmigración en casi un $150 \%$, de manera que se convirtieron, con un $27 \%$, en el primer grupo de procesos federales (Nopper, 2008: 211). Posteriormente, el efecto de la creciente asociación de las acciones contra el terrorismo y de lucha contra la "inmigración ilegal", así como las consecuencias de la aprobación de la USA Patriot Act (2001), aun no teniendo como objetivo la gestión de las migraciones, ha sido la confusión entre el migrante no autorizado y el extranjero enemigo, lo que ha permitido dar una vuelta de tuerca más a la comprensión de su presencia como una amenaza a la seguridad nacional (De Genova, 2006).

En definitiva, la actitud hacia la migración no autorizada y su representación como ilegal ha variado enormemente en Estados Unidos desde el final de la Segunda Guerra Mundial. Los momentos de producción de irregularidad y su posterior regularización se pueden resumir bajo la conocida expresión de política de "puerta giratoria" (Samora, 1971; De Genova, 2004: 189), dado que las sucesivas fases de importación de mano de obra han estado seguidas de fases de deportaciones en masa en las que se han fomentado actitudes negativas hacia la migración no autorizada a partir de distintas instancias. En estas fases proliferan argumentos que intentan justificar la percepción de la inmigración no autorizada como un problema. Por un lado, se encuentra la difícil situación del mercado de mano de obra nacional en fases de recesión de la economía; la competitividad a la hora de acceder a los recursos estatales, a las ayudas sociales y a derechos del Estado de bienestar; y el argumento de la competitividad que se desarrolla en torno a la identidad social de los grupos que conforman el Estado, explicitado en la percepción de la inmigración como una amenaza a la identidad nacional (Calavita, 1998: 149; Nevins, 2002: 93-114). Sin embargo, Joseph Nevins ha señalado que la supuesta "ilegalidad" del migrante no autorizado no se basa en sus características sociales, económicas, políticas o raciales, sino que parte de la asunción de la lógica estatocéntrica por parte de la población en general, es decir, el fundamento de la reacción negativa es un proceso que subyace a dichos argumentos y que es más sutil. La capacidad del Estado de modelar la mentalidad colectiva para pensar la realidad en función de sus propios límites es lo que da lugar, según Nevins (2002: 121), a que el migrante irregular sea identificado como "(...) culpable de una transgresión geográfica por el supuesto crimen de estar en un espacio particular sin autorización del Estado". En este mismo sentido, De Genova (2004: 204) ha criticado que en relación con las políticas de migración y los procesos administrativos de obtención de la nacionalidad la soberanía estatal muestra su carácter marcadamente absolutista, pues la irregularidad de los migrantes sólo es tal en relación con la transgresión de la autoridad soberana del Estado-nación.

\subsection{La producción de irregularidad en Europa occidental}

A continuación se realizará una aproximación a algunos de los procesos de producción de irregularidad que se han dado en distintos Estados de Europa occidental. Estudiar la totalidad de dichos Estados se presenta como una tarea inabordable en un trabajo de estas características. Se hace inevitable una selección geográfica y para ello se propone partir de los casos de Alemania, Francia y Reino Unido. Los tres son países con una larga experiencia en la recepción de migraciones coloniales, laborales y a partir de los años ochenta se han constituido en destino de flujos de refugiados. Otra de las razones que incita a centrar la atención en este área es que la literatura disponible acerca de la gestión de las migraciones es lo suficientemente amplia para abordar a partir de la misma un análisis crítico de los procesos de producción de irregularidad que han acontecido. Así, en primer lugar se propone realizar un breve recorrido del tratamiento de las migraciones no autorizadas desde la Segunda Guerra Mundial a fin de observar cómo se ha llevado a cabo la identificación de flujos bien distintos como "ilegales" o "irregulares" y a partir de ahí exponer una serie de consideraciones sobre las contradicciones que subyacen a tales desarrollos.

Debido a la escasez de mano de obra y a la imperiosa necesidad de acometer la reconstrucción del país, Francia inició tras la Segunda Guerra Mundial procesos de reclutamiento de trabajadores invitados. Éstos llegaron, en un primer 
momento, procedentes de Italia siguiendo los mecanismos que estableció la Oficina Nacional de Inmigración (Bade, 2003: 278). Sin embargo, dicha fórmula se reveló ineficaz en un corto período de tiempo, pues los flujos de trabajadores italianos se redirigieron hacia paises que ofrecian mejores condiciones de trabajo, como Bélgica, Holanda, Suiza o la RFA, y los empresarios franceses recurrieron a la contratación de trabajadores portugueses y españoles que llegaban sin visado o únicamente con visado de turista pero con la intención de trabajar ${ }^{14}$. En la década de los sesenta se produjo, además, la llegada de trabajadores de Turquía y Yugoslavia ${ }^{15}$. Debido a la demanda del mercado de trabajo y a la percepción social positiva de la migración, que se comprendía como una contribución al bienestar de la sociedad (Wihtol de Wenden, 1990: 27), la migración que no llegaba con la documentación y los permisos requeridos no fue conceptualizada, al menos hasta finales de los años sesenta, como migración "irregular" o "ilegal" sino bajo la expresión "migraciones espontáneas" o "migración por sus propios medios". Si bien una ordenanza aprobada en 1945 definía la migración no autorizada como un delito sujeto a penas de cárcel, la demanda de fuerza de trabajo extranjera dio lugar a que se aprobase en 1956 un procedimiento en función del cual se pudiese regularizar la situación de los trabajadores extranjeros cuando contasen con empleo y con una nómina (Düvell, 2010: 298). La regularización de los flujos de trabajadores migrantes no autorizados se desarrolló de forma ordinaria y habitual una vez que se encontraban en el territorio nacional. Es paradójico el hecho de que, si bien en términos jurídicos los inmigrantes eran básicamente españoles, polacos y portugueses, fue la migración colonial argelina, considerada menos désirable, la que desató reacciones más enconadas por parte de la opinión pública francesa y fue la migración en torno a la cual se desarrolló el debate social y político sobre la llegada de extranjeros a Francia (Weil, 1991: 64).

El ambiente favorable hacia la presencia de inmigración no controlada y de trabajadores extranjeros en general empezó a quebrarse antes de la crisis económica de principios de los años setenta. A finales de los años sesenta la inmigración se convirtió en objeto de debate público y político, cuando afloró en la sociedad francesa una creciente preocupación por el acceso de los migrantes a los derechos y beneficios sociales limitados, como la sanidad y la educación, pero también por la escasez de vivienda. Las primeras medidas restrictivas en materia migratoria se aprobaron en 1972 y 1973, momento en el que se suspendieron los procedimientos de regularización y se intentó aplicar la ley de inmigración de forma más estricta (Castles, 1986: 764; Rist, 1979: 97; Weil, 1991: 74; Wihtol de Wenden, 1990: 34). La aprobación de las circulares Marcellin y Fontanet en 1972 ha sido calificada por Düvell como la medida que dio lugar a la transformación del trabajo no reglado en ilegal mediante la penalización de los trabajadores extranjeros que no estuviesen declarados (Düvell, 2010: 298). Podría irse más allá calificándola como la inauguración del proceso que dio lugar a la irregularización de las migraciones no autorizadas que en décadas anteriores eran bienvenidas. En julio de 1974 se puso fin al programa de reclutamiento y a partir de ese momento se intentó cerrar las fronteras mediante la aprobación de nuevas legislaciones restrictivas. La finalidad de estas normativas era poner fin a la relativa laxitud con la que había sido regulada la inmigración hasta el momento y someterla a un control más exhaustivo, pero en todo caso las migraciones se siguieron realizando, ahora bajo la forma de reagrupaciones familiares o migraciones no autorizadas.

El tratamiento que se dio a la migración no autorizada también evolucionó en la República Federal Alemana desde una postura relativamente flexible en los momentos de crecimiento económico hasta una imposibilidad de regularización cuando la situación del mercado laboral empeoró (Sanz Díaz, 2004: 24). Junto a los trabajadores invitados (Gastarbeiter) que llegaron a través de las oficinas de reclutamiento que se abrieron en los países del sur de Europa, se produjo también la llegada de trabajadores que no habían conseguido el visado correspondiente o que sólo contaban con el visado de turista pero que igualmente emprendieron el viaje hacia el norte de Europa (Castles, 1986: 764). Wihtol de Wenden (1990: 27) ha dado cuenta de cómo una vez en destino resultaba relativamente sencillo encontrar trabajo y alojamiento y cómo tras un período de tiempo se podía conseguir regularizar la situación del migrante. En todo caso, jurídicamente siempre se exigió que los trabajadores extranjeros poseyesen un permiso de residencia y trabajo (Castles, 1986: 769).

A través del programa de reclutamiento de trabajadores invitados se intentó que la mano de obra extranjera desempeñase la función de un ejército industrial de reserva para los momentos de crecimiento económico y que se pudiese prescindir fácilmente en momentos de depresión, 
como efectivamente sucedió en la recesión de 1966-1967 (Brubacker, 1992: 171; Castles y Miller, 2004: 94; Sanz Díaz, 2004: 25). Sin embargo, cuando se clausuraron los programas de reclutamiento en 1973 se hizo evidente que este objetivo no se había alcanzado ${ }^{16}$. Se interrumpió la contratación en origen pero una parte importante de los trabajadores extranjeros no retornaron a sus países. El efecto de estas medidas fue que la migración turca, la última en llegar, saliese peor parada, pues contaba con mayor porcentaje de trabajo no autorizado. A diferencia del resto de Estados europeos, la RFA no contempló la posibilidad de poner en marcha procesos de regularización extraordinarios, dado que se consideraba que no harian más que agravar el creciente problema al fomentar nuevas entradas de migrantes indocumentados (Wihtol de Wenden, 1990: 40).

De la misma forma que en el caso francés, el supuesto bloqueo de las fronteras alemanas de los años setenta en realidad no fue tal, pues se permitió la entrada de migrantes que llegaban en forma de refugiados ${ }^{17}$ y en forma de reagrupaciones familiares. El cambio de la naturaleza de las migraciones también generó procesos de ampliación de la irregularidad migratoria porque con la ley aprobada en 1993 sólo podrian optar al status de asilado quienes hubiesen accedido a Alemania sin pasar por un país seguro $y$, tal y como observa Klaus Bade (2003: 320): "(...) Alemania consiguió estar completamente rodeada por este tipo de estados por el simple procedimiento de declararlos seguros". De esta forma los refugiados que Ilegasen legalmente por vía terrestre tenían de antemano negado el status de asilado. La única forma de optar a dicho status en el Estado alemán, si no se cuentan con los medios económicos suficientes para llegar en avión, sería violando las leyes de acceso al territorio. De esta forma la irregularidad migratoria que en un primer momento estaba asociada únicamente a los trabajadores migrantes extranjeros (excluyendo convenientemente a los trabajadores comunitarios) se amplió para asumir también aquellos refugiados a quienes legalmente no se les dejaba otra vía de acceso para conseguir protección.

El caso de Reino Unido difiere de forma notable con respecto al alemán o al francés, pues la identificación de determinadas entradas en su territorio como "ilegales" no ha sido resultado de la clausura de grandes programas de reclutamiento de mano de obra, sino que ha sido fruto de un proceso bastante singular en el cual se han ido estableciendo progresivamente límites a la entrada de determinados ciudadanos británicos, aquellos provenientes de las colonias $^{18}$ (Couper y Santamaria, 1984; Joppke, 1999: 100). Las migraciones coloniales han sido las que han marcado el ritmo de limitaciones en la entrada y también las que han puesto en marcha distintas medidas que finalmente han resultado en la irregularización de flujos migratorios a través de la ampliación de los supuestos que se consideraban infracciones a la ley. Además, como efecto del debate generado en los años sesenta y setenta la migración y la raza se han unido inextricablemente en el imaginario social británico y las palabras "inmigrante" y "persona de color" se han convertido en sinónimos (Castles y Kosack, 1984: 23; Dummet, 2004: 107).

Siguiendo a Couper y Santamaria (1984), la primera limitación que se impuso a los migrantes de la Commonwealth se derivó de la aplicación de la Commonwealth Immigrants Act (1962), en la que se establecía que los migrantes podrían ser sometidos a examen por la policía de inmigración para determinar si su entrada era finalmente permitida. Esta medida no tuvo consecuencias cruciales, pues si en las 24 horas siguientes a la entrada en el territorio no se denegaba el acceso al migrante, éste no podría ser expulsado. El cambio más significativo que introducia la ley fue que sobrepasar el tiempo establecido por el permiso de residencia quedaba recogido como infracción por la cual se podría deportar al migrante. Hay que señalar que los ciudadanos irlandeses, principal mano de obra extranjera en el Reino Unido y considerados deseables y asimilables, fueron convenientemente excluidos de la aplicación de esta ley (Hayter, 2004: 47).

En 1965 se aprobó el libro blanco titulado Immigration from the Commonwealth que, aunque no ilegalizaba la práctica de entrar sin control, introdujo una primera cuña al hablar de evasion from control y alertaba del creciente número de llegadas que se producian en estas circunstancias. Durante este período aparecieron las primeras referencias en el discurso público a la "inmigración ilegal" (Couper y Santamaria, 1984: 446; Düvell, 2010: 298), si bien en los documentos oficiales a partir de los años setenta se utilizaba la expresión illegal entrant.

A finales de los años sesenta se aprobó la Commonwealth Immigrants Act (1968) y con ella se extendieron los su- 
puestos bajo los cuales los migrantes de la Commonwealth cometían un delito al entrar en el territorio, pues pasaba a ser obligatorio presentarse ante la policía de inmigración para ser sometido a examen al llegar al Reino Unido. Si se incumplía este trámite y eran interceptados en los 28 días siguientes, su expulsión podría ejecutarse, ampliando así el plazo anterior de 24 horas. En cualquier caso, una vez superado este período la migración no era considerada "ilegal", aun cuando hubiese accedido de forma clandestina.

La aprobación de la Immigration Act de 1971 inauguró la llamada Dark Age of obsessive control (Bade, 2003: 255). Una de las medidas más importantes que se impuso fue suprimir el límite temporal de 28 días que la ley anterior había fijado para poder identificar una entrada no autorizada. Por otro lado, la entrada no autorizada pasaba a ser definida como ilegal y la nueva normativa recogía por primera vez una referencia explícita a los illegal entrants, que englobaban las personas que entraban, buscaban entrar 0 ya habian entrado ilícitamente en el Reino Unido violando una orden de expulsión (Couper y Santamaria, 1984: 441). El continuo proceso de inflación de la categoría de inmigrante ilegal no quedó ahí, sino que prosiguió durante la década de los setenta extendiéndose a las entradas que se realizaban con documentación falsa, que omitían información necesaria a la policía de inmigración o que se producian de forma clandestina. Pero las medidas que se pusieron en marcha no fueron exclusivamente legales y tampoco estaban centradas únicamente en el control de acceso al territorio: se crearon nuevos tipos de controles para poder acceder a los derechos sociales, de manera que se puede entender que los controles situados anteriormente en las fronteras exteriores del Reino Unido, en sus controles fronterizos, se reprodujeron en el interior del Estado para resolver la ineficacia de los primeros.

En los años ochenta y noventa las dificultades impuestas al acceso por las restrictivas leyes de inmigración y extranjería de los países del norte de Europa redirigieron buena parte de los flujos migratorios laborales hacia los paises del sur de Europa, concretamente España, Grecia, Italia y Portugal, donde a partir de este momento se pueden identificar procesos de irregularización de los flujos migratorios ${ }^{19}$.

En definitiva, en el período de los años sesenta y setenta la producción de regularidad e irregularidad se vinculó en
Francia y la RFA a la gestión de flujos de trabajadores extranjeros, mientras que en el Reino Unido se desarrolló una imagen racializada de la irregularidad a partir de la llegada de ciudadanos británicos de las colonias. Estos procesos de irregularización no han sido fijos o estáticos, sino que han estado sometidos a evolución, de manera que en las décadas posteriores se han extendido a distintos supuestos y se han ampliado a nuevos sujetos, como refugiados y extranjeros en régimen de turista que desempeñaban una actividad laboral. La modificación del tipo de movilidad que es considerada no autorizada (trabajadores extranjeros, refugiados, ciudadanos de las colonias, etc.) pone de manifiesto cómo la regularización o irregularización es un mecanismo en manos del Estado que se pone en marcha de forma distinta en función de las tensiones que se quieran atajar, y de ahí su carácter inestable. Puede ser resultado de las contradicciones que emanan de la lógica expansiva capitalista y la lógica territorial del Estado o puede ser efecto también de las contradicciones entre la lógica nacionalista de carácter exclusivo y la lógica imperialista que de una u otra manera quiere seguir manteniendo lazos de unión con las zonas de influencia. En este sentido, la tesis que ha elaborado De Genova para el caso estadounidense acerca de que la producción de irregularidad es coyuntural y de que no es el resultado necesario de la estructura fija de las sociedades capitalistas (De Genova, 2004: 193) se puede hacer extensiva a los ejemplos europeos planteados, con la salvedad de que no sólo se expresa el interés de mantener permanentemente un trabajo migrante disponible, sino que revelan otras contradicciones consustanciales a los Estados capitalistas.

\section{REFLEXIÓN FINAL}

El hecho de que buena parte de los trabajos especializados en migraciones irregulares presenten de forma recurrente gran interés por definir su objeto de estudio y el hecho de que ninguna de las respuestas ofrecidas satisfaga a la audiencia revela que el problema que se plantea va mucho más allá de la denominación y los contenidos atribuidos a tal irregularidad. Sólo recientemente se ha puesto de manifiesto que es más fértil y pertinente preguntarse cómo se produce la irregularidad migratoria, es decir, que la tarea a desarrollar se enfoque al estudio de los procesos mediante los cuales determinados flujos 
son conceptualizados como ilegales. Esta estrategia sirve además para lograr un objetivo más ambicioso: evitar que las migraciones irregulares se conciban como procesos autónomos.

A la luz de este planteamiento se ha sostenido que, si bien una aproximación analítica que fije una terminología y unos contenidos es necesaria para clarificar y delimitar la investigación a realizar, es imprescindible atender a la dimensión temporal y geográfica de tales factores, pues no son estáticos y se configuran además de forma distinta en cada sociedad. Las continuas referencias a la "inmigración ilegal" en el ámbito internacional han contribuido a pensarla como si tuviese un significado fijo y reconocible con independencia de los ordenamientos legales de cada pais $y$, en definitiva, como si tuviese sentido seguir hablando de migración no autorizada más allá de la esfera estatal. A través del repaso de los casos de Estados Unidos, Reino Unido, Francia y Alemania se ha puesto de manifiesto que la movilidad identificada en cada Estado y en cada período como regular o irregular no remite siempre a un mismo contenido. Se ha mantenido que la producción de esta irregularidad a partir de distintos tipos de movilidad es un mecanismo mediante el cual se intentan ajustar las tensiones existentes entre el impulso expansivo e imperialista de las economías capitalistas y el intento de los Estados de seguir manteniendo un adentro y un afuera, tanto en términos territoriales como de membresía. En este sentido, la supuesta irregularidad del migrante no responde únicamente a la asunción de la lógica estatocéntrica por parte de la opinión pública, tal y como han apuntado distintos académicos, sino que la identificación de su movilidad como no autorizada es un mecanismo -como lo puede ser su regularización- para atajar las contradicciones apuntadas. En definitiva, las definiciones analíticas de las migraciones no autorizadas han de concebirse como meras tentativas y deben ser matizadas mediante la explicitación del contexto social, histórico y legal en el que se generan, pues de otra manera se contribuye a naturalizar o cosificar la irregularidad migratoria.

\section{NOTAS}

* El presente trabajo se ha realizado en el marco del Proyecto de Investigación Políticas migratorias, justicia y ciudadanía (HUM2006-1703/FISO), financiado por el Plan Nacional I+D+i. Agradezco los comentarios a versiones anteriores de este artículo de Fernando Bayón, Carmen Domenech, Marta Irurozqui, Silvia Marcu, Cristina Santamarina, Lorenzo Peña, Michael Janoschka, Juan Carlos Velasco y José Antonio Zamora.

1 En este trabajo se propone la utilización de los términos migrante y migración en lugar de inmigrante 0 inmigración. Las razones que sustentan el empleo de estas expresiones son: a) la búsqueda de unos parámetros que salven el escollo del punto de vista exclusivo de los Estados de acogida y de los flujos migratorios organizados desde una perspecti- va teleológica, en la medida en la que han sido concebidos como flujos unidireccionales que llegan para asentarse y residir en el nuevo Estado, obviando la circularidad de la migración; b) el intento de superar la lógica estatocéntrica con la que se han estudiado tradicionalmente los flujos migratorios, introduciendo así la perspectiva del país de acogida y la experiencia transnacional (Suárez Navaz, 2005). En cualquier caso se tiene presente que en el estudio de la irregularidad migratoria existe desde el principio una perspectiva que de inmediato remite al Estado de acogida, pues es quien establece las condiciones de entrada (López Sala, 2003: 162). Como ha señalado Fargues, en el análisis de la irregularidad migratoria se impone el punto de vista del Estado receptor sobre todo el proceso migratorio (Fargues, 2009: 545).
Aceptado: 24 de febrero de 2010 
2 A lo largo de este artículo se hará una distinción entre los conceptos migrante o migración irregular, por un lado, y el concepto irregularidad migratoria, por otro. Utilizo este segundo en el mismo sentido en el que Nicholas De Genova ha propuesto migrant "illegality" (2002: 421-423), como condición sociopolítica y como status jurídico que implica una relación con el Estado y cuya configuración concreta varía en función de cada momento socio-histórico. Apelo a la referencia de la regularidad y no la ilegalidad por las razones que se explicitan en el primer apartado de este artículo.

3 Pese a que se aboga por la utilización de otra terminología que no haga referencia a la ley, se emplearán los términos migración "ilegal" o "ilegalidad" cuando sea la forma en la que se haya definido la migración no autorizada en un determinado Estado en un periodo histórico. En cualquier caso, la expresión irá entrecomillada para problematizar el uso que se hace de la misma.

4 Cabría señalar que dentro de las múltiples etiquetas con las que se designa a los migrantes no autorizados se encuentran grupos tan diversos y que son considerados irregulares por factores tan distintos como: migrantes en tránsito, trabajadores irregulares, menores descendientes de padres en situación irregular y que heredan el status irregular aunque nunca hayan cruzado una frontera, familiares reagrupados sin autorización, solicitantes de asilo a quienes se les ha denegado dicho status, residentes extranjeros que han perdido el status regular, etc.

5 Ordenanza 45-2658 de 2 de noviembre de 1945 relative aux conditions d'entrée et de séjour en France des étrangers et portant création de I'Office national d'immigration.

6 En Estados Unidos la designación de extranjeros como no deseables es previa a la existencia de legislación estatal que regulase las migraciones internacionales. Con anterioridad a 1860 las autoridades locales estadounidenses desempeñaban la labor de control de la movilidad a fin de mantener el orden público. Estos controles cualitativos definían como migrantes no deseables a criminales, enfermos que pudiesen suponer un riesgo para la salud pública, pobres y minusválidos y grupos marginados por su etnia o raza (Neuman, 2005; Nevins, 2002: 100; Lee, 2002: 3-4; Zolberg, 2005: 195-196). A partir de la aprobación de la Chinese Exclusion Act en 1882 se definió la entrada de inmigración no deseada como una actividad criminal y se inició el proceso legal de exclusión de los trabajadores extranjeros de origen asiático (Lee, 2002: 3). La migración no deseada ya presente en el territorio nacional fue, por otro lado, identificada como inasimilable, en el sentido de que no podian acceder a la ciudadanía.

7 Las expresiones elegible e inelegible alien así como legitimate e illegitimate alien se emplearon a finales del siglo XIX y principios del XX en Estados Unidos para designar a aquellos extranjeros a los que se negaba el acceso a la ciudadanía, como ocurría con la mayor parte de los migrantes que procedian de Asia (Ngai, 2003: 49).

8 Debido al interés del sector industrial del suroeste del país, que requería mano de obra barata, se venía aplicando desde la aprobación de la Immigration Act de 1917 una excepción para que los trabajadores mexicanos no tuviesen que pagar la head tax, ni realizar el test de alfabetismo, además se les eximió del requisito de cumplir los requisitos de las contract labor laws (Samora, 1971: 38).

9 Desde finales de los años 1920 se pusieron en marcha distintas medidas para facilitar la regularización de los migrantes que debido a las nuevas legislaciones eran considerados ilegales. La Registry Act de 1929 permitía legalizar el status de quienes residiesen en el pais desde 1921 tras la paga de una tasa, pero el $80 \%$ de quienes se acogieron a este procedimiento eran europeos. Los mexicanos o no podian pagar la tasa impuesta, 0 no llegaron a conocer la ley, o de conocerla, no la entendieron. Las medidas administrativas que se aprobaron para suspender las deportaciones se aplicaron en su mayoría a inmigrantes europeos. Y por último, el procedimiento de pre-examination era un mecanismo desarrollado para que los inmigrantes sin visado viajasen a Canadá y pudiesen volver posteriormente a Estados Unidos con su situación legal regularizada. Al igual que las medidas anteriores la pre-examination fue aplicada casi exclusivamente a los europeos y canadienses (Ngai, 2003: 73-91).

10 Si bien el Programa Bracero se aprobó en un primer momento como una medida extraordinaria en el marco de la Segunda Guerra Mundial, finalmente se prorrogó hasta 1964 (Massey y Liang, 1989: 203; Samora, 1971: 19).

11 Aunque esta terminología se extendió a partir de los años cuarenta, la expresión se venía utilizando desde los años veinte y designaba los migrantes no autorizados que habían llegado a Estados Unidos desde México cruzando el Río Grande. Posteriormente su 
uso se amplió para designar a todos los migrantes irregulares mexicanos en general (Samora, 1971: 6).

12 La producción de ilegalidad en el Estado moderno como estrategia para establecer control y disciplina social ha sido uno de los análisis de Foucault que más eco han tenido. Según dicho autor: "la existencia de una prohibición legal crea en torno suyo un campo de prácticas ilegales sobre el que se logra ejercer un control y un provecho de elementos también ilegales pero convertidos en manejables por su organización delincuencial" (Foucault, 2005: 285). Este planteamiento ha sido rescatado por De Genova (2002) para el estudio de la irregularidad migratoria y su funcionalidad a la hora abastecer de trabajadores indocumentados al mercado de trabajo. La condición espacializada de la ilegalidad migratoria en tanto que reproducción de las fronteras físicas en el interior del Estado y su relación con la "deportabilidad" dan lugar al paradójico proceso de inclusión a través de la incapacidad de acceder a un status de residencia legal.

13 En los años noventa se pusieron en marcha algunas de las operaciones en la frontera que más eco han tenido como la operación Hold the line en El Paso, Texas, anteriormente denominada Blockade (Dauvergne, 2008: 156), la operación Gatekeeper en California en la zona de San Diego y la operación Safeguard en Arizona (Nevins, 2002: 134-138).

14 El fracaso de la política de reclutamiento de la ONI se puso de manifiesto en que el número de trabajadores no documentados con respecto al número total de trabajadores migrantes pasó del $26 \%$ en 1948 al $82 \%$ en 1968 (Bade, 2003: 279-280;
Castles, 1986: 763; Dummet, 2004: 150; Wintol de Wenden, 1990: 34).

15 De forma paralela a los flujos de migraciones estrictamente laborales Francia experimentó la llegada de migrantes de las colonias, fundamentalmente de Argelia, Marruecos, Túnez, las indias occidentales y del África occidental (Castles, 1986: 764). Tras los procesos de independencia de las colonias se reconoció a los argelinos el derecho de entrada en el territorio francés, pero no ocurrió lo mismo con los migrantes procedentes de otras regiones, que siguieron llegando, pero bajo la forma de migraciones no controladas (Wihtol de Wenden, 1990: 33).

16 Hay que tener en cuenta que para esta fecha el proceso de cierre de fronteras a los trabajadores extranjeros ya se había tornado selectivo, pues quienes procediesen de los países que conformaban la Comunidad Europea podían acceder al territorio de la República Federal Alemana.

17 La República Federal Alemana tenía la política de asilo más aperturista de Europa y hacia ella se dirigieron la mayoría de los solicitantes de asilo procedentes de África y Asia en los años ochenta. La cifra continuó aumentando con la llegada de solicitantes procedentes de Europa del Este a lo largo de esta década y sobre todo a partir de la disolución de Yugoslavia (Bade, 2003: 296 y ss.).

18 En 1948, la British Nationality Act creó por primera vez el status de ciudadano británico, que fue conferido a los 800 millones de personas que hasta entonces habian sido consideradas súbditos del Imperio Británico. A partir de entonces gozarian de libertad de movimiento para entrar en el Reino Unido sin estar sometidas a ningún tipo de control fronterizo o migratorio (Couper y Santamaria, 1984: 438; Joppke, 1999: 101).

19 No es posible detenerse en un estudio exhaustivo de estos procesos, en cualquier caso se apuntarán los factores que propiciaron su desarrollo. Por un lado, la integración de los paises del sur de Europa en la Comunidad Europea y la transformación de sus economías y mercados les convirtió progresivamente en nuevos destinos para los flujos migratorios (Calavita y Suárez, 2003). Otro factor destacable que fomentó la llegada de migraciones a los países del sur de Europa y que determinó que se configurasen como migraciones irregulares es la existencia en todos ellos de un importante sector de economía sumergida, que facilita la inserción laboral de los migrantes no autorizados gracias a su carácter flexible (Sassen, 1999: 105; Reyneri, 2003). Además, las políticas migratorias vigentes no facilitaban la entrada de trabajadores migrantes de forma regular, pues no habian sido diseñadas pensando en la inmigración, sino más bien en fases inmediatamente anteriores en las que era prioritaria la atención a la emigración. Una vez que se inició la reforma y actualización de las legislaciones para cumplir con las condiciones que imponía formar parte de la Comunidad Europea, incluidas las relativas al acceso al territorio nacional, el nuevo perfil que adquirieron las políticas migratorias fue marcadamente restrictivo. La consecuencia inmediata de las nuevas legislaciones de extranjería y de la imposición de visados para buena parte de los Estados que no pertenecian a la CE fue la producción de irregularidad en el ámbito de las migraciones. 


\section{BIBLIOGRAFÍA}

Bade, K. (2003): Europa en movimiento: las migraciones desde finales del siglo XVIII hasta nuestros días, Barcelona, Crítica.

Böcker, A. (ed.) (1998): Regulation of migration: international experiences, Amsterdam, Het Spinhuis.

Brotherton, D. y Kretsedemas, P. (ed.) (2008): "Open Markets, Militarized Borders? Immigration Enforcement Today" en Brotherton, D. y Kretsedemas, P. (ed.): Keeping out the other: A critical introduction to immigration enforcement today, Nueva York, Columbia U. P., pp. 1-28.

Brubaker, R. (1994): Citizenship and Nationhood in France and Germany, Cambridge, Harvard U. P.

Bustamante, J. (1977): "Undocumented Immigration from Mexico: Research Report", International Migration Review, 11(2), pp. 149-177.

Caestecker, F. (1998): "The Changing Modalities of Regulation in International Migration within Continental Europe, 1870-1940", en Böcker, A. (ed.), Regulation of Migration, Amsterdam, Het Spinhuis.

Caestecker, F. (2000): Alien policy in Belgium, 1840-1940. The Creation of Guest Workers, Refugees and Illegal Aliens, Nueva York y Oxford, Berghahn Books.

Calavita, K. (1992): Inside the state: the bracero program, immigration, and the I.N.S., Nueva York, Routledge.

Calavita, K. (1995): "Mexican Immigration to the USA: The Contradictions of Border Control", en Cohen, R. (ed.), The Cambridge Survey of World Migration, Cambridge, Cambridge U. P. pp. 236-244.

Calavita, K. (1998): "Immigratioin Policymaking: Contradictions, myths, and backlash", en Böcker, A. (ed.): Regu- lation of migration: international experiences, Amsterdam, Het Spinhuis, pp. 139-158.

Calavita, K. (2005): Immigrants at the margins: law, race, and exclusion in Southern Europe, Cambridge y Nueva York, Cambridge University Press.

Calavita, K. y Suárez Navaz, L. (2003): "Spanish Immigration Law and the Construction of Difference: Citizens and 'Illegals' on Europe's Southern Border" en Perry, R. W. y Maurer, B. (eds.): Globalization under Construction: Governmentality, Law and Identity, Minneapolis y Londres, University of Minnesota Press, pp. 99-127.

Castles, S. (1986): "The Guest-Worker in Western Europe - An Obituary", International Migration Review, 20(4), pp. 761-778.

Castles, S, y Kosack, G. (1984): Los trabajadores inmigrantes y la estructura de clases en la europa occidental, México, FCE.

Castles, S. y Miller, M. (2004): La era de la migración: Movimientos internacionales de población en el mundo moderno, México, Universidad Autónoma de Zacatecas.

Couper, K. y Santamaria, U. (1984): "An Elusive Concept: The Changing Definition of Illegal Immigrant in the Practice of Immigration Control in the United Kingdom", International Migration Review, 18(3), pp. 437-452.

Coutin, S. B. (2000): Legalizing moves: Salvadoran immigrants' struggle for U.S. Residency, Ann Arbor, University of Michigan Press.

Dauvergne, C. (2008): Making people illegal: What Globalization means for Migration and Law, Nueva York, Cambridge U. P.

De Genova, N. (2002): "Migrant "Illegality" and Deportability in Everyday Life", Annual Review of Anthropology, 31, pp. 419-447.
De Genova, N. (2004): "La produzione giuridica dell'illegalità: Il caso dei migranti messicani negli Stati Uniti", en Mezzadra, S. (ed.), I confini della libertà: per un'analisi politica delle migrazioni contemporanee, Roma, DeriveApprodi, pp. 181-215.

De Genova, N. (2006): "La 'ilegalidad' migratoria y la metafísica del antiterrorismo: los 'derechos de los inmigrantes' como secuelas del Estado de Seguridad Nacional" extraído de http://www.rebelion.org/noticia. php?id=41021

De Genova, N. y Ramos-Zayas, A. (2003): Latino Crossings: Mexicans, Puerto Ricans, and the Politics of Race and Citicienship, New York and London, Routledge.

Dummet, M. (2004): Sobre inmigración y refugiados, Madrid, Cátedra.

Düvell, F. (2008): "Clandestine migration in Europe", Social Science Information, 47(4), pp. 479-497.

Düvell, F. (2010): "Irregular Migration: A global, historical and economic perspective", en Vertovec, S. (ed.), Migration, Abingdon, Routledge, vol. 2, pp. 289-310.

Fargues, P. (2009): "Work, Refuge, Transit: An Emerging Pattern of Irregular Immigration South and East of the Mediterranean", International Migration Review, 43 (3), 544-577.

Gutiérrez, D. (1995): Walls and mirrors: Mexican Americans, Mexican immigrants and the politics of ethnicity, San Francisco, University of California Press.

Hayter, T. (2004): Open Borders: The Case Against Immigration Controls, Londres, Pluto Press.

Heckmann, F. (2004): "Illegal Migration: What Can We know And What Can We Explain? The Case of Germany", International Migration Review, 38 (3), pp. 1103-1125. 
Joppke, C. (1999): Inmigration and the Nation-State: The United States, Germany and Great Britain, Oxford, Oxford U. P.

Koser, K. (2005): "Irregular migration, state security and human security", extraido de http://www.humansecurity gateway.com/showRecord. php?Recordld $=19282$

Lee, E. (2002): "Enforcing the Borders: Chinese Exclusion along the U.S. Borders with Canada and Mexico, 1882-1924", The Journal of American History, 89(1), pp. 54-86.

López Sala, A. M. (2003): "La inmigración irregular en la investigación sociológica", en Godenau, D. y Zapata Hernández, V. M. (eds.): La inmigración una aproximación interdisciplinar, Tenerife, Observatorio de la Inmigración en Tenerife, pp. 161-184.

Massey, D. S. y Liang, Z. (1989): "The long term consequenes of a temporary worker program: the US Bracero experience", Population Research and Policy Reviwe, 8(3), pp. 199-226.

Mezzadra, S. (ed.) (2004): I confini della libertà: per un'analisi politica delle migrazioni contemporanee, Roma, DeriveApprodi.

Neuman, G. (2005): "Qualitative Migration Controls in the Antebellum United States", en Fahrmeir, A.; Faron, 0. y Weil, P. (eds.), Migration Control in the North Atlantic World, Nueva York y Oxford, Berghahn Books, pp. 106119.

Nevins, J. (2002): Operation Gatekeeper: The Rise of the "illegal Alien" and the Making of the U.S.-Mexico Boundary, Nueva York y Londres, Routledge.

Ngai, M. (1999): "The Architecture of Race in American Immigration Law: A Re- examination of the Immigration Act of 1924", Journal of American History, 86 (1), pp. 67-92.

Ngai, M. (2003): "The Strange Career of the Illegal Alien: Immigration Restriction and Deportation Policy in the United States, 1921-1965", Law and History Review, 21(1), disponible en http:// www.historycooperative.org/journals/ lhr/21.1/ngai. html

Ngai, M. (2004): Impossible Subjects: illegal aliens and the making of modern America, Princeton, Princeton U. P.

Nopper, T. (2008): "Why Black Immigrants Matter: Refocusing the Discussion on Racism and Immigration Enforcement", en Brotherton, D. y Kretsedemas, P. (ed.): Keeping out the other: A critical Introduction to Immigration Enforcement Today, Nueva York, Columbia U. P., pp. 204-237.

Reyneri, E. (2003): "Illegal Immigration and the Underground Economy" extraído de http://hdl.handle.net/1885/41778

Rist, R. C. (1979): "Migration and Marginality: Guestworkeres in Germany and France", Daedalus, 108(2), pp. 95-108.

Samora, J. (1971): Los Mojados, the Wetback Story, Notre Dame y Londres, University of Notre Dame Press.

Sanz Díaz, C. (2004): "Clandestinos", "ilegales", "espontáneos"...: La emigración irregular de españoles a Alemania en el contexto de las relaciones hispano-alemanas, 1960-1973, Madrid, Comisión Española de Historia de las Relaciones Internacionales.

Sassen, S. (1999): Guests and Aliens, Nueva York, The New Press.

Schrover, M.; Van der Leun, J.; Lucassen, L. y Quispel, C. (2008): "Introduction: Illegal migration and gender in a global and historical perspective", en Schrover, M. et al. (ed.), Illegal migration and Gender in a Global and Historical Perspective, Amsterdam, Amsterdam U.P., pp. 9-38.

Stoddard, E. R. (1976): "A conceptual Analysis of the "Alien Invasion": institutionalized Support of Illegal Mexican Aliens in the U.S.", International Migration Review, 10(2), pp. 157-189.

Strozza, S. (2004): "Estimates of the illegal foreigners in Italy: a review of the literature", International Migration Review, 38(4), pp. 309-331.

Suárez Navaz, L. (2005): "Ciudadanía y migración: ¿un oximoron?", en Cuadernos del Observatorio de las migraciones y la Convivencia Intercultural de la Comunidad de Madrid (OMC), n. ${ }^{\circ}$ 4, pp. 29-47.

Van Ejil, C. (2008): "Tracing back 'illegal aliens' in the Netherlands, 18501940", en Schrover, M. et al. (ed.): Illegal migration and Gender in a Global and Historical Perspective, Amsterdam, Amsterdam U.P., pp. 39-56.

Weil, P. (1991): La France Et Ses Étrangers: L'aventure D'une Politique De L'inmigration 1938-1991, Paris, Calmann-Lévy.

Wihtol de Wenden, C. (1990): "The Absence of Rights: the Position of Illegal Immigrants", en Layton-Henry, Z. (ed.): The Political Rights of Migrant Workers in Western Europe, Londres, Sage Publications, pp. 27-47.

Zolberg, A. (2005): "The Archaelogy of 'Remote Control'", en Fahrmeir, A.; Faron, 0. y Weil, P. (eds.), Migration Control in the North Atlantic World, Nueva York y Oxford, Berghahn Books, pp. 195-222. 